\title{
A STRONG LAW OF LARGE NUMBERS FOR MARTINGALES ${ }^{1}$
}

\author{
SHEY SHIUNG SHEU AND YU SHAN YAO
}

\begin{abstract}
We derive a moment inequality for the Skorohod representation theorem and apply it to obtain a strong law of large numbers for martingales.
\end{abstract}

1. Introduction. In the early sixties, Skorohod proved the following result; see Skorohod [5, p. 163], Hall and Heyde [4, p. 269].

THEOREM 1. Let $\left\{S_{n}=\sum_{j=1}^{n} X_{j}, n \geq 1\right\}$ be a zero mean martingale. There exists a probability space supporting a standard Brownian motion $\{B(t)\}$ and a sequence of nonnegative random variables $\left\{\tau_{n}, n \geq 1\right\}$ such that

(i) $\left\{B\left(\sum_{j=1}^{n} \tau_{j}\right)\right\}$ has the same joint distribution as $\left\{S_{n}\right\}$,

(ii) $E\left(\tau_{n}^{r}\right) \leq C_{r} E\left(\left|X_{n}\right|^{2 r}\right), C_{r}$ is a constant depending on $r, r \geq 1$.

In this paper, we shall derive an inequality more general than Theorem 1(ii) and apply this inequality to prove a strong law of large numbers for martingales.

2. A moment inequality. We shall adopt the following representation method which is slightly different from the one given by Freedman [3, p. 68]. Let $X$ be a zero mean random variable with distribution function $F$, let $f(x)=F^{-1}(x)=$ $\inf \{t: F(t) \geq x\}, 0 \leq x \leq 1$. Let $g(x)$ be a monotone solution of the function equation $\int_{x}^{g(x)} f(t) d t=0$. Now construct a probability space supporting a standard Brownian motion process $\{B(t)\}$ and a random variable $U$ which is independent of $\{B(t)\}$ and uniformly distributed over $[0,1]$. Conditioning on $U=x$, define $\tau(x)=\inf \{t: B(t)=f(x)$ or $f(g(x))\}$. Then the distribution of $B_{\tau}$ is $F$ and $E(\tau)=E\left(X^{2}\right)$. To see this, let $G(u, v)$ be the distribution which assign probability $|u| /(|u|+|v|)$ at $v$ and probability $|v| /(|u|+|v|)$ at $u, u v<0$. Note that conditioning on $U=x, B_{\tau}$ has distribution $G(f(x), f(g(x)))$. Therefore, if $\psi$ is a bounded measurable function, then

$$
E \psi\left(B_{\tau}\right)=\int_{0}^{1}[\psi(f(x))|f(g(x))|+\psi(f(g(x)))|f(x)|](|f(g(x))|+|f(x)|)^{-1} d x
$$

Using the facts that

$$
g(g(x))=x, \quad f(x)=f(g(x)) g^{\prime}(x)
$$

Received by the editors May 4, 1983 and, in revised form, August 25, 1983.

1980 Mathematics Subject Classification. Primary 60G40, 60G42.

Key words and phrases. Brownian motion, Skorohod's representation, strong law of large numbers, martingale.

${ }^{1}$ Research supported by the National Sciences Council, Republic of China, Grant NSC71-0204M007-14. 
we see

Hence

$$
\begin{aligned}
\int_{0}^{1} \psi(f(g(x)))|f(x)|(|f(x)|+|f(g(x))|)^{-1} d x \\
\quad=\int_{0}^{1} \psi(f(x))|f(x)|(|f(x)|+|f(g(x))|)^{-1} d x .
\end{aligned}
$$

$$
E \psi\left(B_{\tau}\right)=\int_{0}^{1} \psi(f(x)) d x=\int_{-\infty}^{\infty} \psi(x) d F(x) .
$$

That is, $B_{\tau}$ has distribution $F$. By the same reason,

$$
E(\tau)=\int_{0}^{1}|f(x) f(g(x))| d x=\int_{0}^{1} f^{2}(x) d x=\int_{-\infty}^{\infty} x^{2} d F(x)=E\left(X^{2}\right) .
$$

Moreover, we shall show

THEOREM 2. Let $\phi(x), x \geq 0$, be a nonnegative function such that for some $a$, $b, \frac{1}{2}<a<b<\infty, \phi(x) / x^{a}$ is nondecreasing and $\phi(x) / x^{b}$ is nonincreasing. Then $E \dot{\phi}(\tau) \leq C E \phi\left(X^{2}\right)$, where the constant $C$ depends only on $a$ and $b$.

Proof. Following Breiman [1], let $T=\inf \{t: B(t)=u$ or $v\},-u \leq 0 \leq v$. Then

$$
P(T \geq t)=\frac{4}{\pi} \sum_{n=1}^{\infty}(2 n+1)^{-1} \exp \left[-\frac{\lambda_{n}^{2} t}{2 r^{2}}\right] \sin \left(\frac{\lambda_{n} u}{r}\right),
$$

where $\lambda_{n}=(2 n+1), r=u+v$. By induction, $\sin \left(\lambda_{n} u / r\right) \leq 4 \pi^{2}(2 n+1) u v / r^{2}$. Hence,

$$
\begin{aligned}
E \phi(T) & =\int_{0}^{\infty} P(T \geq t) d \phi(t) \\
& \leq C_{1} \frac{u v}{r^{2}} \int_{0}^{\infty}\left(\sum_{n=1}^{\infty} \exp \left[-\frac{\lambda_{n}^{2} t}{2 r^{2}}\right]\right) d \phi(t) .
\end{aligned}
$$

From the inequality

$$
\begin{aligned}
\sum_{n=0}^{\infty} e^{-\lambda_{n}^{2} s} & \leq e^{-8 s} \sum_{n=0}^{\infty} e^{-4 n^{2} \pi^{2} s} \\
& \leq e^{-8 s}\left(1+\int_{0}^{\infty} e^{-4 \pi^{2} s x^{2}} d x\right)=\left(1+\frac{C_{2}}{\sqrt{s}}\right) e^{-8 s}
\end{aligned}
$$

follows

$$
E \phi(T) \leq C_{3}\left[\frac{u v}{r^{2}} \int_{0}^{\infty} \exp \left[-\frac{4 t}{r^{2}}\right] d \phi(t)+\frac{u v}{r} \int_{0}^{\infty} \exp \left[-\frac{4 t}{r^{2}}\right] \frac{d \phi(t)}{\sqrt{t}}\right]
$$

Write

where

$$
\int_{0}^{\infty} e^{-s t} d \phi(t)=\int_{0}^{1 / s} e^{-s t} d \phi(t)+\int_{1 / s}^{\infty} e^{-s t} d \phi(t)
$$

$$
\begin{aligned}
\int_{0}^{1 / s} e^{-s t} d \phi(t) & =e^{-1} \phi\left(\frac{1}{s}\right)+s \int_{0}^{1 / s} e^{-s t} \phi(t) d t \\
& \leq e^{-1} \phi\left(s^{-1}\right)+s^{a+1} \phi\left(s^{-1}\right) \int_{0}^{1 / s} t^{a} e^{-s t} d t \\
& \leq C_{4} \phi\left(s^{-1}\right)
\end{aligned}
$$


and

$$
\begin{aligned}
\int_{1 / s}^{\infty} e^{-s t} d \phi(t) & =-e^{-1} \phi\left(s^{-1}\right)+s \int_{1 / s}^{\infty} e^{-s t} \phi(t) d t \\
& \leq-e^{-1} \phi\left(s^{-1}\right)+s^{b+1} \phi\left(s^{-1}\right) \int_{1 / s}^{\infty} t^{b} e^{-s t} d t \\
& \leq C_{5} \phi\left(s^{-1}\right) .
\end{aligned}
$$

Similarly

$$
\begin{aligned}
\int_{0}^{\infty} t^{-1 / 2} e^{-s t} d \phi(t) & =\int_{0}^{1 / s} t^{-1 / 2} e^{-s t} d \phi(t)+\int_{1 / s}^{\infty} t^{-1 / 2} e^{-s t} d \phi(t) \\
& \leq C_{6} \sqrt{s} \phi\left(s^{-1}\right),
\end{aligned}
$$

where $C_{1}, C_{2}, C_{3}, C_{4}, C_{5}, C_{6}$ are constants and $C_{4}, C_{5}$ depend on $a, C_{6}$ depends on $b$. Therefore, one concludes

$$
E \phi(T) \leq C \frac{u v}{r^{2}} \phi\left(\frac{r^{2}}{4}\right) \leq C \frac{u v}{r^{2}} \phi\left(\frac{u^{2}+v^{2}}{2}\right),
$$

where $C$ is a constant depending on $a$ and $b$.

Note

$$
\phi\left(\frac{1}{2}\left(u^{2}+v^{2}\right)\right) \leq \max \left\{\phi\left(u^{2}\right), \phi\left(v^{2}\right)\right\} \leq \phi\left(u^{2}\right)+\phi\left(v^{2}\right) .
$$

Hence,

This implies

$$
E \phi(T) \leq C \frac{u v}{r^{2}}\left(\phi\left(u^{2}\right)+\phi\left(v^{2}\right)\right)
$$

$$
\begin{aligned}
E \phi(\tau) & \leq C \int_{0}^{1} \frac{|f(x) f(g(x))|}{(|f(x)|+|f(g(x))|)^{2}}\left[\phi\left(f^{2}(x)\right)+\phi\left(f^{2}(g(x))\right)\right] d x \\
& =C \int_{0}^{1}|f(x)| \phi\left(f^{2}(x)\right)(|f(x)|+|f(g(x))|)^{-1} d x \\
& \leq C \int_{0}^{1} \phi\left(f^{2}(x)\right) d x=C E \phi\left(X^{2}\right) . \quad \text { Q.E.D. }
\end{aligned}
$$

3. A strong law. Using the result in Theorem 2, we shall prove a strong law of large numbers for martingales. First, observe a simple fact: if two sequences of random variables $\left\{X_{n}\right\}$ and $\left\{Y_{n}\right\}$ have the joint distribution, $C$ is a constant, then $P\left(\left|X_{n}-C\right| \geq \varepsilon\right.$, i.o. $)=P\left(\left|Y_{n}-C\right| \geq \varepsilon\right.$, i.o. $) \forall \varepsilon>0$; therefore, $X_{n} \rightarrow C$, a.s. if and only if $Y_{n} \rightarrow C$, a.s.

THEOREM 3. Let $S_{n}=\left\{\sum_{j=1}^{n} X_{j}, n \geq 1\right\}$ be a zero mean martingale. Let $\phi(x), x \geq 0$, satisfy the assumptions in Theorem 2 and $\phi(x+y) \leq \phi(x)+\phi(y)$, for all $x, y \geq 0$. If for some sequence of positive numbers $\left\{a_{n}\right\}$, we have $a_{n} \uparrow \infty$ and $\sum_{n=1}^{\infty} E \phi\left(X_{n}^{2}\right) / \phi\left(a_{n}\right)>\infty$, then $S_{n} / a_{n}^{p} \rightarrow 0$ a.s. for every $p>\frac{1}{2}$.

PROOF. Let $\left\{B\left(\sum_{j=1}^{n} \tau_{j}\right)\right\}$ represent $\left\{S_{n}\right\}$ as in Theorem 1. By Theorem 2 and the assumption that $\sum_{n=1}^{\infty} E \phi\left(X_{n}^{2}\right) / \phi\left(a_{n}\right)<\infty$, we conclude

$$
\sum_{n=1}^{\infty} \phi\left(\tau_{n}\right) / \phi\left(a_{n}\right)<\infty \quad \text { a.s. }
$$


Applying Kronecker's Lemma we get

$$
\frac{1}{\phi\left(a_{n}\right)}\left(\sum_{j=1}^{n} \phi\left(\tau_{j}\right)\right) \rightarrow 0 \text { a.s. }
$$

By the assumption $\phi(x+y) \leq \phi(x)+\phi(y)$, we see

$$
\phi\left(\sum_{j=1}^{n} \tau_{j}\right) / \phi\left(a_{n}\right) \rightarrow 0 \quad \text { a.s. }
$$

Because $\phi(x) / x^{a}$ is nondecreasing and $\phi(x) / x^{b}$ is nonincreasing, we have

$$
\phi\left(\sum_{j=1}^{n} \tau_{j}\right) / \phi\left(a_{n}\right) \geq \min \left\{\left[\frac{1}{a_{n}}\left(\sum_{j=1}^{n} \tau_{j}\right)\right]^{a},\left[\frac{1}{a_{n}}\left(\sum_{j=1}^{n} \tau_{j}\right)\right]^{b}\right\} .
$$

Therefore,

$$
\frac{1}{a_{n}}\left(\sum_{j=1}^{n} \tau_{j}\right) \rightarrow 0 \text { a.s. }
$$

Without loss of generality, we may assume $S_{n} \rightarrow \infty$ a.s. which implies $\sum_{j=1}^{n} \tau_{j} \rightarrow \infty$ a.s. But the law of the iterated logarithm for Brownian paths tells us

$$
B\left(\sum_{j=1}^{n} \tau_{j}\right) /\left(\sum_{j=1}^{n} \tau_{j}\right)^{p} \rightarrow 0 \quad \text { a.s. } \forall p>\frac{1}{2} .
$$

Hence,

$$
S_{n} / a_{n}^{p} \rightarrow 0 \text { a.s. } \forall p>\frac{1}{2} \text {. Q.E.D. }
$$

COROLlARY 4. Let $\left\{S_{n}=\sum_{j=1}^{n} X_{j}\right\}$ be a zero mean martingale. If $E\left(X_{n}^{2}\right)<$ $\infty \forall n$, and $s_{n}^{2}=\sum_{j=1}^{n} E\left(X_{j}^{2}\right) \rightarrow \infty$, then $S_{n} / s_{n}^{p} \rightarrow 0$, a.s. $\forall p>1$.

Proof. Choose $\phi(x)=x, a_{n}=s_{n}^{2}\left(\log s_{n}^{2}\right)^{1+\varepsilon} \uparrow \infty, \varepsilon>0$. Then

$$
\sum_{n=1}^{\infty} \frac{E \phi\left(X_{n}^{2}\right)}{\phi\left(a_{n}\right)}=\sum_{n=1}^{\infty} \frac{E\left(X_{n}^{2}\right)}{s_{n}^{2}\left(\log s_{n}^{2}\right)^{1+\varepsilon}}<\infty
$$

by Dini's theorem, and consequently

$$
S_{n} / s_{n}^{2 p}\left(\log s_{n}^{2}\right)^{p+p \varepsilon} \rightarrow 0 \quad \text { a.s. for every } p>\frac{1}{2}
$$

which implies

$$
S_{n} / s_{n}^{p} \rightarrow 0 \text { a.s. for every } p>1 \text {. Q.E.D. }
$$

COROLlaRY 5. Let $\left\{S_{n}=\sum_{j=1}^{n} X_{j}\right\}$ be a zero mean martingale. If

$$
\sum_{n=1}^{\infty} E\left|X_{n}\right|^{r} / n^{r}<\infty, \quad 1<r \leq 2,
$$

then $S_{n} / n^{p} \rightarrow 0$ a.s. $\forall p>1$. 
ProOF. Choose $\phi(x)=x^{r / 2}, a_{n}=n^{2} \uparrow \infty$. Apparently, $\phi(x)$ satisfies the required conditions in Theorem 3 and

$$
\sum_{n=1}^{\infty} \frac{E \phi\left(X_{n}^{2}\right)}{\phi\left(a_{n}\right)}=\sum_{n=1}^{\infty} E\left|X_{n}\right|^{r} / n^{r}<\infty
$$

by assumption. Hence $S_{n} / n^{p} \rightarrow 0$ a.s. for every $p>1$. Q.E.D.

REMARK 6. Chow [2] has shown that if $\sum_{n=1}^{\infty} E\left(X_{n}^{2}\right) / n^{2}<\infty$, then $S_{n} / n \rightarrow 0$ a.s.

\section{REFERENCES}

1. L. Breiman, On the tail behavior of sums of independent random variables, Z. Wahrsch. Verw. Gebiete 9 (1967), 20-25.

2. Y. S. Chow, On a strong law of large numbers for martingales, Ann. Math. Statist. 38 (1967), 610.

3. D. Freedman, Browmian motion and diffusion, Holden-Day, San Francisco, Calif., 1971.

4. P. Hall and C. C. Heyde, Martingale limit theory and its applications, Academic Press, New York, 1980.

5. A. V. Skorohod, Studies in the theory of random processes, Addison-Wesley, Reading, Mass., 1965.

Institute of ApPlied Mathematics, National TSing HuA UNiVersity, Hsinchu, TAIWAN 300, REPUBLIC OF CHINA

Current address (S. S. Sheu): Department of Statistics, University of California, Berkeley, California 94720

Current address (Y.S. Yao): Department of Business Mathematics, Soochow University, Taipei, Taiwan, Republic of China 